\title{
ENDOMETRIAL CARCINOMA AND THE IMMUNE SYSTEM - NEW INSIGHTS BASED ON THE RESEARCH LITERATURE
}

\author{
Kameliya Tsvetanova ${ }^{1}$, Slavcho Tomov², Grigor Gorchev ${ }^{2}$ \\ ${ }^{1}$ UMHAT "Dr. G. Stranski" Ltd. - Pleven, Clinic of Anaesthesiology and Intensive Care \\ ${ }^{2} U M H A T$ "Dr. G. Stranski” Ltd. - Pleven, Clinic of Gynecologic Oncology, \\ Medical University - Pleven
}

\begin{abstract}
The aim of this paper is to perform a review of the research literature on the issue of endometrial cancer and the impact on the immune system of the two major surgical methods - open and minimally invasive.
\end{abstract}

Keywords: endometrial cancer, robotic surgery, cytokine profile

Corpus uteri cancer is the most frequently occurring carcinoma among females in Europe. Furthermore, it is among the ten most common cancers in general.

Despite the progress of the diagnostic and therapeutic methods, the number of cancer cases and mortality is on the rise worldwide. This increase is related both to the higher diagnostic capacities and to the increased frequency of triggering pathogenetic mechanisms associated with modern lifestyles: overweight, hypokinesia, metabolic and hormonal disorders. (1) According to data from the Bulgarian National Cancer Registry, corpus uteri cancer accounts for $8.3 \%$ share of all malignant diseases among females and ranks third in terms of incidence after breast and colon cancer (Fig. 1), $(2,3)$.

Address for correspondence:

Kameliya Tsvetanova

Clinic of Anaesthesiology, Reanimation and Intensive Care,

Oncology Center, University Hospital - Pleven,

8 A, Georgi Kochev Blvd

5800 Pleven, Bulgaria

e-mail: kamelia_tsvetanova@abv.bg

Received: June 27, 2016

Accepted: August 16, 2016

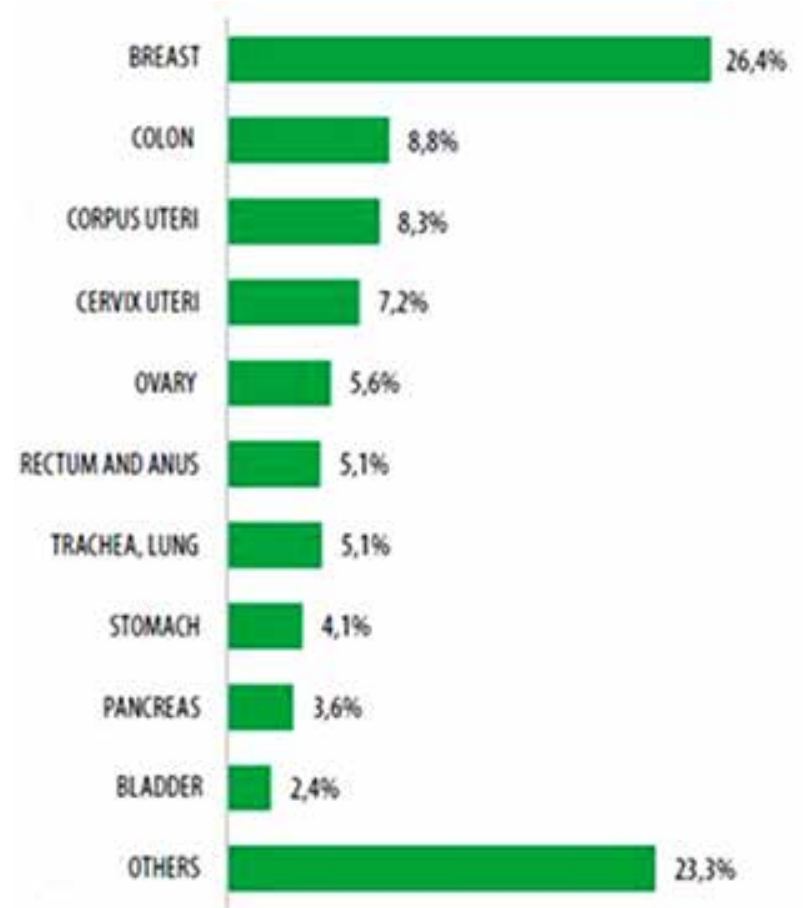

Figure 1. Percentage of distribution of the most frequent malignancies among females in Bulgaria, $2012(4,5)$.

The reasons for its development are various. One of the leading among them is obesity.

Back in the 1980s. of the past century Gambrell et al. (1983), and Davis et al. (1981) demonstrated that 
patients whose weight is $50 \mathrm{~kg}$ over their normal limit are exposed to a higher risk of developing endometrial carcinoma $(6,7)$.

The source of elevated circulation levels of estrogen in patients with obesity is the aromatization of androstenedione to estrone, which takes place in the peripheral adipose tissue (8).

Other major factors for the development of endometrial carcinoma include diabetes mellitus, family history, high-fat diet, etc. $(9,10)$.

Corpus uteri carcinomas fall into two main categories:

Type I - endometroide type, hormone dependent. It is preceded by endometrial hyperplasia, with slow development and good prognosis;

Type II - clear-cell, serous, etc.; non-hormone dependent, evolving against a background of atrophic endometrium; aggressive in its progressing, formating early metastases, with poor prognosis.

The histopathological subtypes of endometrial carcinoma are:

A. Endometrial carcinoma:

Papillary;

Secretory;

Cilliary

Adenocarcinomawith squamous differentiation;

B. Metastatic carcinoma

C. Serous carcinoma

D. Undifferentiated carcinoma

E. Squamous cell carcinoma

F. Clear cell carcinoma

G. Combined cell carcinoma

H. Mucinous carcinoma

Among these, adenocarcinoma is the most frequent histological type.

It is distinguished by considerable distortion of the glands' arrangements, their number is growing, and various grades of atypia are observed. The overall picture is characterized by a decrease in stroma, neoplastic growth of the glands, which are located "back to back“.

Cell proliferation leads to stratification, papillary formation and eventually, to gland obliteration. The individual cellular changes include varying degrees of immaturity and dedifferentiation of cells, hyperchromatosis, aniso-chromatosis, monstrous nuclei, and various numbers of mitoses. These signs are united under the name "pleomorphism“ (11).

The clinical manifestation of the disease is characterized by:

$\otimes$ Genital bleeding that occurs against a background of endometrial hyperplasia - an early symptom such as vaginal bleeding within or beyond the menstrual cycle;

$\bowtie$ Pain in the lower abdomen - occurring much later, when the tumor process is advanced;

$\otimes$ Fluor - late symptom associated with necrotic changes in the tumor.

As with any other disease, corpus uteri carcinoma affects the immune system.

It is known that it consists of two separate subdivisions: non-specific or innate immune system and adaptive, or specific immune system. Each subdivision has cellular and humoral elements allowing the immune system to defend the body against foreign cancer and pathogens.

Surgical intervention related to a major surgical trauma causes development of „hormonal stress response", which is achieved by the systematic release of cortisol, catecholamines, angiotensin, vasopressin, insulin, etc., and cytokine production as well during the acute-phase response. In the human body, there is a sensitive balance between pro- and anti-inflammatory cytokines. As a result of the surgical trauma, the lowered immune response quickly entails the development of the so-called Syndrome of Systemic Inflammatory Response (SIRS) and multiple organ failure (MOF).

This raises the question of choosing the most sparing surgical technique whereby the frequency of these dangerous complications can be prevented or reduced.

The past decade has seen the publication of many studies regarding the impact of the two traditional surgical methods: open and minimally invasive on various functional immune parameters.

Based on these, it may be inferred that big surgical operations lead to a period of cell-mediated immunosuppression, which may exert an influence on the patient's recovery.

Most researchers share the common view that laparotomy causes increased secretion of cytokines and respiratory mediatory burst. 
In minimally invasive surgical methods, the extent to which the $\mathrm{CO} 2$-induced pneumoperitoneum leads to suppression of the function of macrophages is certain. It is also clear that open surgery is related to higher levels of CRP and IL- 6 as compared to the minimally invasive surgical methods (12).

The pro-inflammatory cascade activation after infection, after surgery or trauma, is important for the development of subsequent immune dysfunction, susceptibility to multiple organ failure and sepsis.

Although there are conflicting data between the studies in relation to certain immune parameters, there is a general agreement in respect to other variables.

It is demonstrated that additional factors, such as anesthesia and preoperative blood transfusion, pain and hyperglycemia may further distort the immune performance.

A study of Scholl et al. conducted in 2012 describes the manner in which "surgical stress" and inflammatory reactions affect the immune system and how it changes the sensitivity of the patient to these adverse events (13).

A study conducted by Bobocea et al. demonstrates that big conventional surgical operations give rise to a significant change in immunological response, which is especially important in cancer patients, as the postoperative suppression is associated with septic complications, a lower survival rate, tumor spread and metastasis formation.

Clinical studies have shown that laparoscopic surgery preserves in a better way the immunological function of patients. Postoperative plasma concentrations of C-reactive protein (CRP) , IL-10, IL-6 and TNF- $\alpha$ have been demonstrated as being lower after minimally invasive operations (14).

The author comes to the conclusion that immune function is influenced by the degree of surgical trauma. In minimally invasive surgery, reduced acute-phase response is observed as compared to open procedures and it leads to better preservation of the cellular immune mechanisms.

According to Kehlet et al. (1999), the peri-operative immune system response is determined by the severity of the surgical trauma (15).

El Azab et al. (2002), Kawamura et al. (2006), and Cheng (2005) report higher levels of pro-inflam- matory cytokines IL- 8 and IL- 6 as well as of the antiinflammatory cytokine IL-10 $(16,17,18)$.

Kehlet (1999) explains this with the smaller tissue trauma (15).

The research team of Sheeran (1977) describes a significant increase in plasma concentrations of IL-6 only between the second and the fourth post-surgery hour (19).

Other interesting results can be found in the study of Schneemilch (2004), which registers intra and postoperative high levels of IL-10 (20).

In 2014, Maas et al. (2014) publish a randomized trial comparing the conventional and minimally invasive surgery. They establish the existence of a significant difference between the two studied groups of patients in favor of the minimally invasive operating technique, which results in better levels of leukocytes and IL-8 (21).

In regard to CRP and IL- 6 no significant differences between the two groups have been observed. They find that the poor results obtained with the conventional access are due to postoperative respiratory infections observed in the postoperative period.

Another randomized study of surgical stress and postoperative immune function after conventional and minimally invasive surgery is that of Veenhof et al. (2010), who summarize that in minimally invasive surgical access, a better short-term preservation of the immune function is achieved. The increased level of IL- 6 is lower than that in open surgical access (4.6 vs. 10.8; $\mathrm{p}=0.003)$ (22).

No differences have been observed between the two groups in regard to the number of leukocytes, monocytes, CRP, and IL-8 in the postoperative period.

A large-scale study on the impact of robot-assisted and conventional surgery on the immune system in females with endometrial carcinoma is conducted by Dimitrov et al., and its results convincingly prove the immune-sparing role of robotic surgery in these patients (12).

\section{CONCLUSION}

Even though the prognosis of endometrium carcinoma is good in most of the cases, its diagnosting in later stages is associated with high morbidity and mortality level. 
Therefore, prevention, early diagnosis and minimization of surgical intervention comprise the key to successful treatment, reducing the severity of the disease progression, and ultimately contribute to a lesser moral, social and economic cost of the disease.

An increasingly greater number of gynecologists, in addition to the usual clinical and histological studies, extend the information about the neoplastic process so as to include biochemical and immunological markers - tumor markers, hormones, lymphocyte populations, cytokines, lesion-inflammatory process markers, etc.

The comprehensive assessment of the immunological level and the abnormal intracellular metabolism are the basis for individual differences, neoplastic aggression, influence of anesthesia and surgery, postoperative recovery and prognosis.

In the field of gynecological oncology, there is an increasing theoretical and practical interest in the effects of different surgical methods with respect to the immune system as a prognostic factor for the treatment effect on the neoplastic process.

\section{REFERENCES}

1. Dimitrov T. Cytokine profile in women with obesity in stage I of endometrial carcinoma operated by robotic and conventional open surgical intervention. Dissertation paper, 2016. Page 6 (in Bulgarian)

2. Valerianova Z., Bulgarian National Cancer Registry. Sofia, 2014. (in Bulgarian)

3. Dimitrov T. Cytokine profile in women with obesity in stage I of endometrial carcinoma operated by robotic and conventional open surgical intervention. Dissertation paper, 2016. Page 9 (in Bulgarian)

4. Valerianova Z. Malignancies in Bulgaria, the European Union states and North America. $8^{\text {th }}$ National Congress in Oncology with international participation, 2011, 10-13 November, Sofia. Collection of abstracts, $42-43$ (in Bulgarian)

5. Dimitrov T. Cytokine profile in women with obesity in stage I of endometrial carcinoma operated by robotic and conventional open surgical intervention. Dissertation paper, 2016. Page 16 (in Bulgarian)

6. Gambrell RD., BagnellCA, Greenblat RB: Role of estrogenes and progesterone in the etiology and prevention of endometrial cancer. Am J ObstedGynecol 146:696-707,1983.
7. Anderson B, Connor JP, Andrews JI, Davis CS, Buller RE, Sorosky JI, Benda JA. Obesity and prognosis in endometrial cancer. Am J Obstet Gynecol. 1996 Apr;174(4):1171-8; discussion 1178-9.

8. Schenker JG, Weinstei D., Okori E: Estradiol and testosterone levels in the periferal and ovarian circulation in patients with endometrial cancer. Cancer 44: 1809-1812,1997.

9. Sidway MK., Silverberg SG: Endometrial carcinoma: Pathologic factors of therapeutic and prognostic significance. Pathol Ann 27: 153-185, 1992.

10. Gainsberg SB: Current concepts in cancer: The chaning nature of endometrial cancer. N Engl J Med. 302:729-731,1980.

11. Karagyozov I., Doganov N. (rev.) Gynecology, Sofia, Journal of Medicine and Physical Education, 2005. (in Bulgarian)

12. Dimitrov T. Cytokine profile in women with obesity in stage I of endometrial carcinoma operated by robotic and conventional open surgical intervention. Dissertation paper, 2016. Page 75 (in Bulgarian)

13. Scholl R., Bekker A., Babu R. Neuroendocrine and Immune Responses to Surgery. Anesthesiology. 2012 Volume 30 Number 3. Internet Scientific Publications.

14. Bobocea AC, Trandafir B, Bolca C, Cordoş Minimally invasive surgery in cancer. Immunological response. Chirurgia (Bucur). 2012 Mar-Apr;107(2):154-7.

15. Kehlet H. Surgical stress response: does endoscopic surgery coufer an advange? World Jurnal of Surgery 23:801-807.

16. El Azab S.R., Rossel P.M.J., JJ de Langue. E.M. van Wijk and al. Effect of VIMA with sevofluraneversus TIVA with propofol of midazolam-sulfentanyl on the cytokineresponseduring CABG Surgery. European Journal of Anaesthesia. 2002. 19:276-282.

17. Kawamura T., M.Kadusaki N., N. Nara., A. Kaise et al.2006. Effect of Sevofluraneon cytokine balancein patients undergoing coronary after bypas graft syrgery. Journal of Cardthoracic and Vadcuar Anaesthesia 20: 503-508.

18. Cheng C.R., 2005. Inflamatory response to anesthesia and way to attenuate it. Advvances in Anesthesia 23:107-141.

19. Sheernan P., Hall G.M. 1997. Cytokines in anaesthesia. British Journal of Anaesthesia 78: 201-219. 
20. Schneemilch CE, Schilling T. Effects of general anaesthesia on inflammation. Best Pract and Res Clin Anaesthesiology 2004; 18: 493-507

21. Maas KW., Biere SS., van Hoonq IM, van der Peet DL. Cuesta MA.Immunnological changes sfter minimally invasiveor conventional esophageal resecttionfor cancer: a randomized trial. World J Surg 2014 Jan. 38(1):131-7.

22. Veenhof AA., Sietses C, vonBloomberg BM. Van Hooqstraren IM. Vd Pas MH. Et al. The surgical stress response and postoperative immune function after laparoscopic or conventional total mesorectal excision in rectal cancer: a randomized trial. Int J Colorectal Dis. 2011 Jan; 26 910 53:9. 